\title{
Angiogenesis after plasmid VEGF 165 gene transfer in an animal model
}

\author{
JAN SKÓRA ${ }^{l}$, PIOTR BARĆl, TOMASZ DAWISKIBA ${ }^{l}$, DAGMARA BACZYŃSKA ${ }^{2}$, \\ AGNIESZKA MASTALERZ-MIGAS ${ }^{3}$
}

${ }^{1}$ Department of Vascular, General and Transplantation Surgery, Wroclaw Medical University, Poland

${ }^{2}$ Molecular Techniques Unit, Wroclaw Medical University, Poland

${ }^{3}$ Department of Family Medicine, Wroclaw Medical University, Poland

\begin{abstract}
Objectives: This study evaluated the angiogenic efficacy of gene delivery by intramuscular (IM) injections of VEGF 165 plasmid in an animal model.

Material and methods: 36 rats underwent intramuscular injections of plasmid to hind limbs. The control group (18 animals) received empty plasmid. Group 1 received plasmid containing genes for vascular endothelial growth factor (VEGF). After 12 weeks, all the animals were sacrificed and tissue samples from the thigh muscle from hind limbs were harvested for histological and immunohistochemistry examinations.

Results: There were significant differences in the histological findings in the structure of muscle tissue of the hind limbs between two groups. There was evidence of angiogenesis in the samples from group 1. There was no angiogenesis present in the control group. The expression of VEGF 165 protein in the immunohistochemistry examination demonstrated gene delivery to muscles of hind limbs in group 1. Levels of expression of VEGF protein in the control group were not increased. The histological structure of the samples from group 1 confirmed successful gene delivery to muscles of hind limbs.

Conclusions: Intramuscular injections of plasmid of VEGF induced angiogenesis and increased the concentration of VEGF protein in muscle tissue.
\end{abstract}

Key words: angiogenesis, VEGF 165 plasmid, animal model.

(Centr Eur J Immunol 2013; 38 (3): 305-309)

\section{Introduction}

Epidemiological data indicate that frequency of occurrence of the critical lower limb ischemia (CLI) in Europe and the USA range between 500 and 1000 new cases per population of 1 million people [1-3]. CLI often results in the high percentage of patients undergoing amputations and death among patients $[3,4]$. Currently applied pharmacological medication or endovascular procedures in CLI patients yield very poor results. Several studies indicate a death rate of $25 \%$ to $30 \%$ per year in CLI patients who do not qualify for surgery $[1,3]$.

The latest scientific discoveries paved the way for the entirely new concepts and techniques for treatment of the critical lower limb ischemia $[1,5]$. One of the new methods of improving the microcirculation in critically ischemic tissues involves a process of angiogenesis $[5,6]$.
A process involving the growth of new blood vessels is a subject of interest of several research institutes. This vivid interest is augmented not only by the significance of the process in the pathologic physiology of cancer but also by the theoretical possibility of utilising this knowledge in therapy of the ischemic heart disease and lower limb ischemia. Angiogenesis is a process involving blood vessel formation by splitting off the existing ones [5, 6]. It is a very complex process that requires collaboration of numerous cells and cytokines. In angiogenesis, one of the most important cytokines is the vascular endothelial growth factor (VEGF) [5-7]. It is the VEGF that by stimulating the activation, proliferation and migration of epithelial cells initiates formation of vessels in the damaged or ischemic tissue. VEGF plays a key role in angiogenesis and may easily be utilized in the therapeutic pro-

Correspondence: Agnieszka Mastalerz-Migas, Department of Family Medicine, Wroclaw Medical University, Syrokomli 1, 51-141 Wroclaw, Poland, e-mail: agnieszka.migas@gmail.com 
motion of vessel growth in the ischemic tissues (neoangiogenesis) [8].

Therefore, for the reasons specified above, we have decided to evaluate, in an animal model, the effect of the plasmid that includes the VEGF165 protein coding gene.

\section{The aim of the study}

1. Evaluation of the efficacy of the induction of new blood vessel formation by intramuscular injection of the plasmid VEGF165 gene within the hind extremities of the laboratory animals, i.e. Fisher rats.

2. Evaluation of the safety of the VEGF165 gene therapy.

\section{Material and methods}

The research protocol was approved by the Ethics Committee of the Medical University of Wroclaw. Approval No.: KB-926/2003.

Experimental tests were carried out in the Department of Pathomorphology of the Wroclaw Medical University.

For research purposes, the plasmid that includes the human VEGF165 coding gene and an empty control plasmid was used. Gene constructs were provided by the Department of Molecular Technology of the Wroclaw Medical University.

The apyrogenecity of the plasmid was confirmed by a Limulus amebocyte lysate assay, Pyrochrome Chromogenic Test Kit (Charles River) in the Laboratory of Medical Microbiology in the IITD Institute of Immunology of Infectious Diseases of the Polish Academy of Sciences in Wroclaw. Plasmid sterility was confirmed by the Department of Medical Microbiology of the Wroclaw Medical University.

For the purposes of the present study, a group of 36 healthy Fisher rats, weighing from $200 \mathrm{~g}$ to $250 \mathrm{~g}$, was selected and qualified as a research material. All selected animals were approved for the experimental gene therapy by the Commission of Bioethics.

The treatment group or Group 1 consisted of 18 animals that in 4 consecutive intramuscular injections were administered a total plasmid dose of $2 \mathrm{mg}$, with the hind right limb as an injection site.

The control group consisted of practically identical 18 animals who were injected an empty (placebo) control plasmid.

After 12 weeks, all animals were euthanized and their necropsy included taking the muscle tissue samples from the intramuscular injection site for the purpose of analysis. The collected samples were used for the following microscopic examinations:

1. Histopathology

Histologic specimens of the muscle samples were thinly cut into $4 \mu \mathrm{m}$ slices and stained with hematoxylin and eosin. The samples were examined using an Olympus BX50 light microscope.
2. Immunocyto/histochemistry for the detection of VEGF protein

Immunocyto- and histochemical analysis involved paraffin-embedded $4 \mu \mathrm{m}$ tissue sections, mounted on silanized DAKO (DAKO ${ }^{\circledR}$ No. S 3003) glass slides. Next, the samples were deparaffinized with xylene and transferred through the alcohol series of lessening strength down to water.

Antigens in formalin-preserved tissues were retrieved using Target Retrieval Solution DAKO ${ }^{\circledR}$ No. S1700 and then heated for 30 minutes in $90^{\circ} \mathrm{C}$ water bath. Endogenous peroxidase activity was blocked for 10 minutes with a diluted 3\% hydrogen peroxide $\left(\mathrm{H}_{2} \mathrm{O}_{2}\right)$ solution. Next, the slices were stained with primary antibody: VEGF antibody (vascular endothelial growth factor) No. AF-239-NA of RD Systems; dilution factor $1 / 200$, incubation at room temperature for 30 minutes. Next, they were washed in TBS and stained with the biotinylated antibody $\left(\mathrm{SAB}^{\circledR}+\mathrm{Kit}\right.$, DAKO K 0679) for 15 minutes. After TBS washing the slices were incubated for 15 minutes with streptavidin-peroxidase complex $\left(\mathrm{LSAB}^{\circledR}+\right.$ Kit, DAKO K 0679).

The immunocytochemical reaction was caused by 3,3'Diaminobenzidine tetrahydrochloride (DAB + Liquid K 3486 $D A K O)$. The slices were subsequently rinsed in running water and dewatered by alcohol series. The material was xylene overexposed and it was then placed in Canada balsam.

The samples were assessed using an Olympus BX-50 light microscope.

\section{Results}

All rats survived for the full planned 12-week test period of intramuscular plasmid administration. The clinical course was uncomplicated.

\section{Necropsy results}

Necropsy did not reveal any pathological changes in organs and tissues of the animals.

\section{Histological results}

For the purpose of the present study, muscle tissue specimens from the intramuscular plasmid injection site were used. In the control group (empty placebo plasmid), the histological picture of the striated muscles was normal. No neoangiogenesis in microcirculation was observed (Fig. 1).

In group 1 (VEGF 165 plasmid) a normal histological picture, corresponding to the striated muscles was revealed. In all animals, within the microcirculation many endothelial cells and a process of the growth of new blood vessels (neoangiogensis) was found (Fig. 2).

\section{Immunocyto/histochemistry results}

For the purpose of our study, segments of the striated muscles from the intramuscular plasmid injection site were used. In the control group (empty placebo plasmid), the his- 


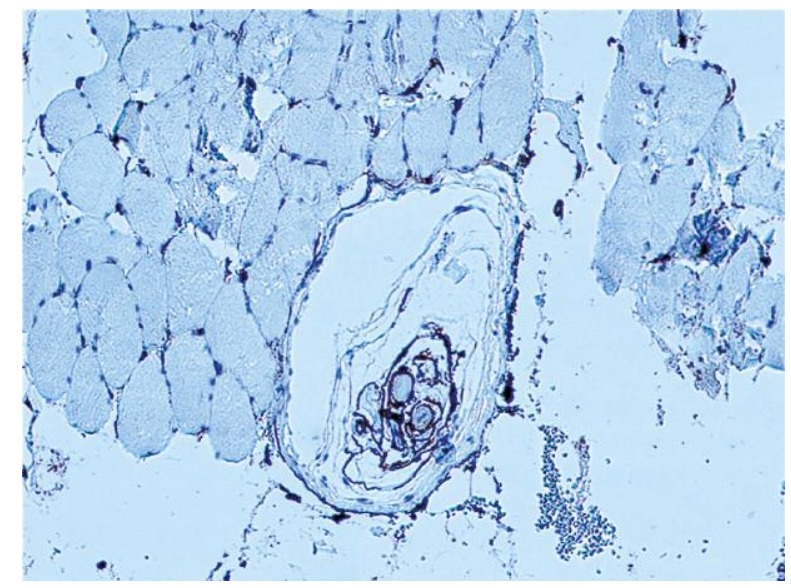

Fig. 1.

tological picture of the striated muscle tissue was normal. In the endothelial cells and extracellular matrix, no increased accumulation of the VEGF165 protein was noted. No formation of new capillaries or increased endothelial cell proliferation was revealed (Fig. 3).

In group 1 (VEGF 165 plasmid), large deposits of the VEGF165 protein were noted both within the extracellular matrix and in the vicinity of the newly formed capillary vessels. All preparations revealed an endothelial cell proliferation. A complete picture reflected the neoangiogenesis (Fig. 4).

\section{Discussion}

In the discussed experimental material, during the full 12week observation period, in 18 animals of the control group

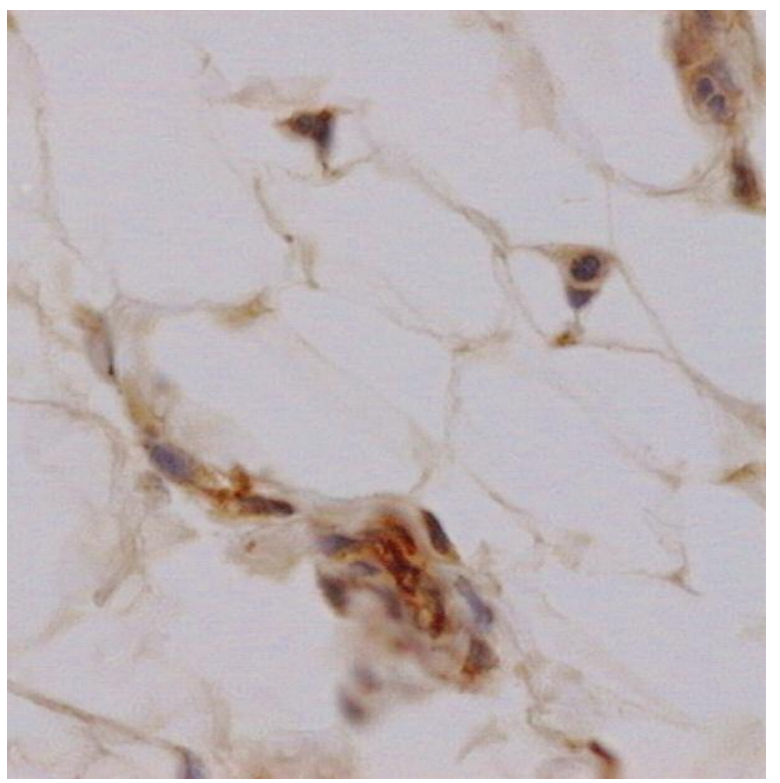

Fig. 3.

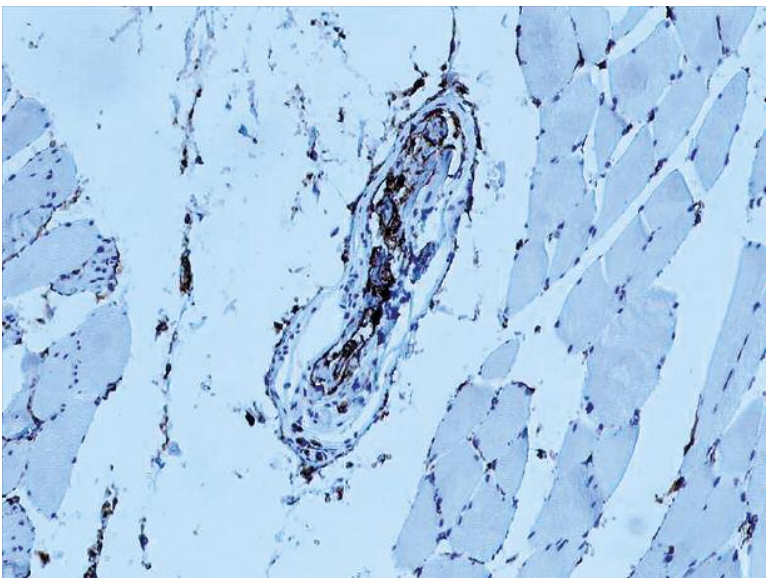

Fig. 2.

(empty plasmid) no clinical complications were observed. Necropsy material revealed no significant pathology.

In group 1, observations carried after the administration of VEGF coding gene plasmid revealed no clinical complications. Similarly, the necropsy material revealed no significant pathology.

Therefore, it can be concluded that plasmid injections do not pose any danger to health or threat to life of the laboratory animals, i.e. Fisher rats.

Lack of changes in the clinical picture is the evidence that the intramuscular injection of empty or the human VEGF165 coding gene plasmid does not significantly influence the basic life functions of the experimental animals.

On the basis of the presented necropsy material, it should be assumed that intramuscular injections of the vascular endothelial growth factor (ph VEGF165) do not promote any general angiogenesis in the internal animal organs, nor stimulate neoplastic processes [8].

Histological and immunocyto/histochemistry results in the control group indicated no significant changes in the tis-

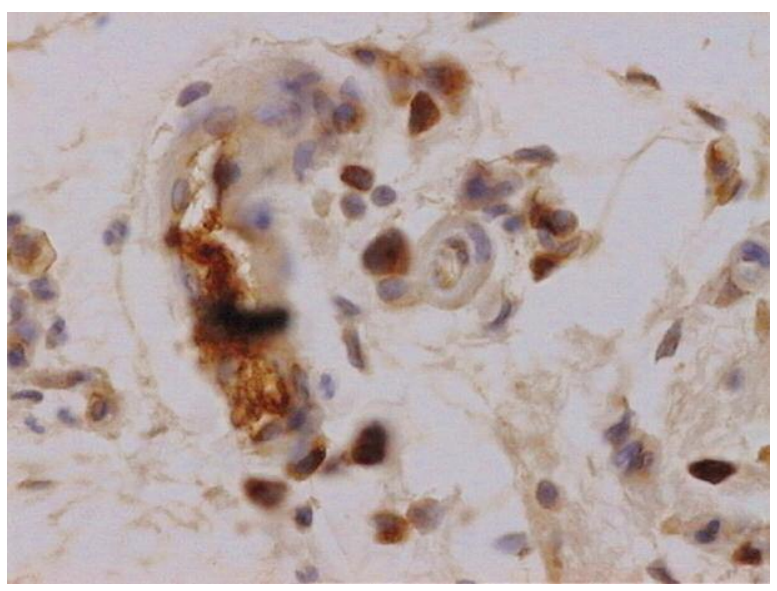

Fig. 4. 
sue structure of all preparations collected from the intramuscular injection site. The obtained results confirm other published experimental studies and indicate that an empty plasmid does not influence the tissue physiology at the injection site [9-12]. Lack of changes in the striated muscle preparations, uncomplicated course of 12-week observation period and the necropsy results demonstrate that an administration of an empty plasmid does not significantly influence the state of health of the experimental animals $[8,9,12]$.

In treatment group 1, in all histological and immunocyto/histochemistry preparations obtained after an application of a VEGF165 plasmid, a promotion of angiogenesis was observed. The phenomenon was evidenced by the new capillaries in the microcirculation, proliferation of endothelial cells and increased deposits of the VEGF165 protein in the plasmid injection site.

At the same time, it should be emphasized that intramuscular injections of the VEGF165 plasmid did not stimulate angiogenesis outside the injection site. This was evidenced by the lack of pathological changes in the necropsy material $[8,9,12]$. The obtained results document the process of angiogenesis only in the sites of administration of the vascular endothelial growth factor (VEGF165) [8, 9, 11, 12].

The growth of new blood vessels (angiogenesis) is a complicated multistage process, involving numerous changes in the overall cellular environment. First, an increased permeability in blood vessels and laying of the extracellular proteins in the matrix $[5,6,11]$ is observed. It is followed by the degrading of the basement membrane, reorganization of the original vessel walls and preciously controlled proteolysis of the matrix molecules [5, 6, 11]. These processes enable and prepare the tissue for the next stage of invasion, migration and proliferation of the endothelial cells upon stimulation by VEGF. The next essential process occurring during the formation of new blood vessels is the organization of multicell aggregates into tubelike structures $[5,6,11]$. This stage occurs only due to the spatial and tridimensional matrix structure and direct cell contact with the extracellular matrix. As a result of this process and VEGF stimulation, the cells change their shape into a more pointed form, better adhering to each other and forming an organisation resembling a blood vessel. The final stage consists in final differentiation into a mature blood vessel and stabilisation $[5,6,11]$. All the cells participating in the formation of the new blood vessel are stimulated by the vascular endothelial growth factor [13-16].

For the reasons specified above and on the basis of the obtained results, it should be assumed that VEGF belongs to proteins determining formation of new veins and arteries. This growth factor was discovered in 1983, as a factor increasing vascular permeability (VPF) and exceeding the maximum response to histamine by 50000 . Later, due to some debate over terminology, it acquired its contemporary name $[13,14]$. Vascular endothelial growth factor is a subfamily of growth factors, which currently includes 6 proteins: VEGF-A, -B, -C, -D, -E and placental growth factor
PIGF [24]. Vascular endothelial growth factor A is the most important and best known one. This gene may be represented in five different forms that differ in the number of amino acids per protein molecule: $121,145,165,189$ or $206[9,14]$. Vascular endothelial growth factor is synthesized by several cell types: endothelial, tumor, macrophages, T lymphocytes, smooth muscle, kidney, keratocytes, astrocytes and osteoblasts [15-18]. The main transcription factor that regulates VEGF cell synthesis is HIF-1, hypoxia inducible factor-1, which stimulates the protein expression in response to insufficient blood supply or cell damage.

The main biological effect of VEGF is modulating endothelial cell biology. The protein regulates also the development of the vascular system [9, 14]. During embryonic development it stimulates the development of aorta and other arteries, as well as of the venous and lymphatic system [14]. Vascular endothelial growth factor is a potent mitogen both for endothelial and smooth muscle cells that participate in formation or reconstruction of arterial vessels [9, 14].

Vascular endothelial growth factor is believed to be a major contributor to initial stages of angiogenesis as it regulates and stimulates the initiation of the process. However, further stages, especially maturity of blood vessels, require collaboration of numerous cells and cytokines [16-18].

While comparing the obtained results to these quoted in the literature on the subject, it should to be assumed that angiogenesis is a multistage process, in which coordinated interaction of extracellular matrix, cells and cytokines is indispensible. Due to the complexity of the process, it is difficult to effectively interfere with formation of new blood vessels solely by administration of the vascular endothelial growth factor [13-15]. The solution that seems to solve this problem is a simultaneous administration of the endothelial growth factors [10, 13-15].

\section{Conclusions}

1. Intramuscular injections of plasmid that includes the VEGF165 coding gene do not pose any danger to health of experimental animals and do not cause any local or general side effects.

2. Intramuscular injection of the plasmid that includes the VEGF165 coding gene does not promote any general angiogenesis or stimulate neoplastic processes in the internal animal organs.

3. A process involving the growth of new blood vessels and increased accumulation of the VEGF165 protein in the injection site of an empty plasmid was observed.

The authors declare no conflict of interests.

\section{References}

1. Minar E (2009): Critical limb ischaemia. Hamostaseologie 29: 102-109. 
2. Jensen SA, Vatten LJ, Myhre HO (2006): The prevalence of chronic critical lower limb ischaemia in a population of 20,000 subjects 40-69 years of age. Eur J Vasc Endovasc Surg 32: 60-65.

3. Norgren L, Hiatt WR, Dormandy JA, et al. (2007): On behalf of the TASC II Working Group. Inter-Society Consensus for the Management of Peripheral Arterial Disease (TASC II). Eur J Vasc Endovasc Surg 33: S1-S75.

4. Mehler PS, Coll JR, Estacio R, et al. (2003): Intensive blood pressure control reduces the risk of cardiovascular events in patients with peripheral arterial disease and type 2 diabetes. Circulation 107: 753-756.

5. Losordo DW, Dimmeler S (2004): Therapeutic angiogenesis and vasculogenesis for ischemic disease, part I: angiogenic cytokines. Circulation 109: 2487-2491.

6. Losordo DW, Dimmeler S (2004): Therapeutic angiogenesis and vasculogenesis for ischemic disease, part II: cell-based therapies. Circulation 109: 2692-2697.

7. Hill JM, Zalos G, Halcox JP, et al. (2003): Circulating endothelial progenitor cells, vascular function, and cardiovascular risk. N Engl J Med 348: 593-600.

8. Shyu KG, Chang H, Wang BW, Kuan P (2003): Intramuscular vascular endothelial growth factor gene therapy in patients with chronic critical leg ischemia. Am J Med 114: 85-92.

9. Anghel A, Mut-Vitcu B, Savu L, et al. (2007): Clinical improvement after treatment with $\operatorname{VEGF}(165)$ in patients with severe chronic lower limb ischaemia. Genomic Med 1: 47-55.

10. Tateishi-Yuyama E, Matsubara H, Murohara T, et al. (2002): Therapeutic angiogenesis for patients with limb ischaemia by autologous transplantation of bone-marrow cells: a pilot study and a randomized controlled trial. Lancet 360: 427.

11. Tongers J, Roncalli JG, Losordo DW (2008): Therapeutic angiogenesis for critical limb ischemia microvascular therapies coming of age. Circulation 118: 9-16.

12. Hao X, Månsson-Broberg A, Grinnemo KH, et al. (2007): Myocardial angiogenesis after plasmid or adenoviral VEGFA(165) gene transfer in rat myocardial infarction model. Cardiovasc Res 73: 481-487.

13. Chen F, Tan Z, Dong CY, et al. (2007): Combination of $\operatorname{VEGF}(165) /$ Angiopoietin-1 gene and endothelial progenitor cells for therapeutic neovascularization. Eur J Pharmacol 568: 222-230.

14. Ylä-Herttuala S, Rissanen TT, Vajanto I, Hartikainen J (2007): Vascular endothelial growth factors: biology and current status of clinical applications in cardiovascular medicine. J Am Coll Cardiol 49: 1015-1026.

15. Kobulnik J, Kuliszewski MA, Stewart DJ, et al. (2009): Comparison of gene delivery techniques for therapeutic angiogenesis ultrasound-mediated destruction of carrier microbubbles versus direct intramuscular injection. J Am Coll Cardiol 54: 1735-1742.

16. Shimamura M, Sato N, Morishita R (2011): Experimental and clinical application of plasmid DNA in the field of central nervous diseases. Curr Gene Ther 11: 491-500.

17. Yasumura EG, Stilhano RS, Samoto VY, et al. (2012): Treatment of mouse limb ischemia with an integrative hypoxiaresponsive vector expressing the vascular endothelial growth factor gene. PLoS One 7(3): e33944.

18. Enestvedt CK, Hosack L, Hoppo T, et al. (2012): Recombinant vascular endothelial growth factor 165 gene therapy improves anastomotic healing in an animal model of ischemic esophagogastrostomy. Dis Esophagus 25: 456-464. 